\title{
Beyond the "hot-and-cold" game: A demonstration of computer-controlled shaping
}

\author{
FRANCISCO J. SILVA \\ University of Redlands, Redlands, California
}

\begin{abstract}
Shaping, or the method of successive approximations, is widely taught in introductory psychology and the psychology of learning as a procedure for establishing new behavior. This article illustrates a computer-controlled shaping demonstration that allows the user to specify several critical parameters of the shaping process and that then shapes the user's mouse movements toward an arbitrary virtual (invisible) target on the computer screen. The relative effectiveness of different shaping parameters can be assessed by examining several dependent measures, such as the distance of the cursor from the target across time and the rate at which reinforcers were earned. This demonstration allows students to move beyond the notion that shaping is simply the application of the "hot-and-cold" game and to understand that there is a science underlying the art of shaping.
\end{abstract}

Shaping, or the method of successive approximations, involves the reinforcement of successively closer approximations to a final target response, while extinguishing previous approximations. Although often thought of as more art than science (e.g., Catania, 1998; Midgley, Lea, \& Kirby, 1989; Pear \& Legris, 1987; Timberlake \& Silva, 1994), there are some observations that experienced shapers are aware of. For example, frequent reinforcement is necessary to keep the organism active, thereby increasing the probability of it emitting a response closer to the target behavior. It may be that reinforcement simultaneously strengthens the response it followed and some degree of behavioral variation (activity) necessary for reinforcement to "select" new responses that are closer to the final target behavior (Antonitis, 1951; Catania, 1998; Donahoe, Burgos, \& Palmer, 1993; Machado, 1997; Stokes, 1995). However, too much reinforcement for a response can lead to the perseveration of that behavior. In these circumstances, reducing the frequency of reinforcement is necessary, but at the risk of inactivity and the extinction of previously established responses.

To demonstrate the process of shaping and some of its challenges, instructors often use the children's "hot-andcold" game, in which a student's behavior is shaped by someone who dispenses feedback by saying "hot" and "cold" when the student is getting closer to or farther from, respectively, emitting the final desired response. Although entertaining, this demonstration does not readily allow students to learn about the science of shaping, and even distorts the concept in at least two ways. First, shap-

I thank two anonymous reviewers and Allen Neuringer for their helpful comments on an earlier version of this article, and Trish Cornez and David Niguidula for their programming suggestions. A copy of the program can be obtained from F.J.S. by sending a Macintosh-formatted disk. Correspondence should be addressed to F. J. Silva, Department of Psychology, University of Redlands, P.O. Box 3080, 1200 East Colton Avenue. Redlands, CA 92373-0999 (e-mail: silva@uor.edu). ing involves the reinforcement of successively closer approximations to a final target response, while extinguishing previous approximations. Although saying "hot" when a student is getting closer to emitting the target response can be considered a positive reinforcer, saying "cold" is not an instance of extinction. Instead, saying "cold" is a punisher that the student will try to avoid; remaining silent when the student is getting farther from the target behavior is an instance of extinction. Second, the person saying "hot" and "cold" often dispenses these reinforcers and punishers in a series of gradations by saying things like "hot," "hotter," "very hot," or "cold," "colder," "very cold." Although there is nothing in the definition of shaping that prohibits using different magnitudes of reinforcement, doing so in the hot-and-cold game makes it more difficult to relate the game to empirical studies (e.g., Eckerman, Hienz, Stern, \& Kowlowitz, 1980; Midgley et al., 1989; Pear \& Legris, 1987; Platt, 1973) and, to a lesser extent, conceptual analyses of shaping (e.g., Catania, 1998; Donahoe et al., 1993; Stokes, 1995). As it has been used in countless studies and illustrated in as many textbooks, the intensity of the reinforcer is held constant during shaping. Thus, rats are shaped to press a lever by reinforcing successive approximations with a pellet of food, not one pellet when the rat orients toward the lever, two pellets when the rat walks toward the lever, three pellets when the rat sniffs the lever, four pellets when the rat raises its paw, and so on. In reality, then, the hot-and-cold game is not a very accurate demonstration of shaping, nor does it permit students in the class to see or understand the variables underlying the procedure. ${ }^{1}$

To provide students with a demonstration of shaping that allows them to see the variables underlying the procedure, I developed a computer-controlled demonstration in which the instructor or a student specifies several critical parameters of the shaping process. The program then uses these values to shape the user's mouse movements toward an arbitrary virtual target on the computer 
screen. This procedure is based closely on that of Pear and Legris (1987), in which a computer-controlled videomonitoring system tracks the position of a pigeon's head and shapes contact of the head with a specific target (a virtual sphere). In their study, the apparatus recorded the Cartesian coordinates of the bird's head 30 times per second as it moved about an experimental chamber. The computer program defined a $3-\mathrm{cm}$ virtual target sphere whose center was located near the lower left rear corner of the chamber. Concentric with the target sphere was a shaping sphere whose radius was programmed to contract by $1 \mathrm{~cm}$ when it was contacted by the bird's head. Each contact with the shaping sphere resulted in reinforcement, which consisted of brief access to food. For every $10-\mathrm{sec}$ period in which the bird did not contact the shaping sphere, its radius expanded by $0.25 \mathrm{~cm}$. This process repeated itself until the bird contacted the target sphere, at which time the shaping sphere was programmed not to expand. Reinforcement now occurred only when the bird contacted the target sphere. A schematic of this procedure is illustrated in Figure 1.

Thus, according to Pear and Legris's (1987) method, five variables that are controlled by the experimenter are presumed to affect the shaping process: (1) the size of the radius of the target sphere, (2) the distance the shaping sphere contracts when it is contacted by the animal, (3) the amount of time permitted to pass without a contact with the new radius of the shaping sphere before its radius expands, (4) the distance that the radius of the shaping sphere expands, and (5) the frequency with which the position of the animal is sampled.

The purpose of the computer-controlled demonstration described in this article is to show students how each of these variables can affect the process and outcome of shaping. For instance, it should be easier to contact a larger

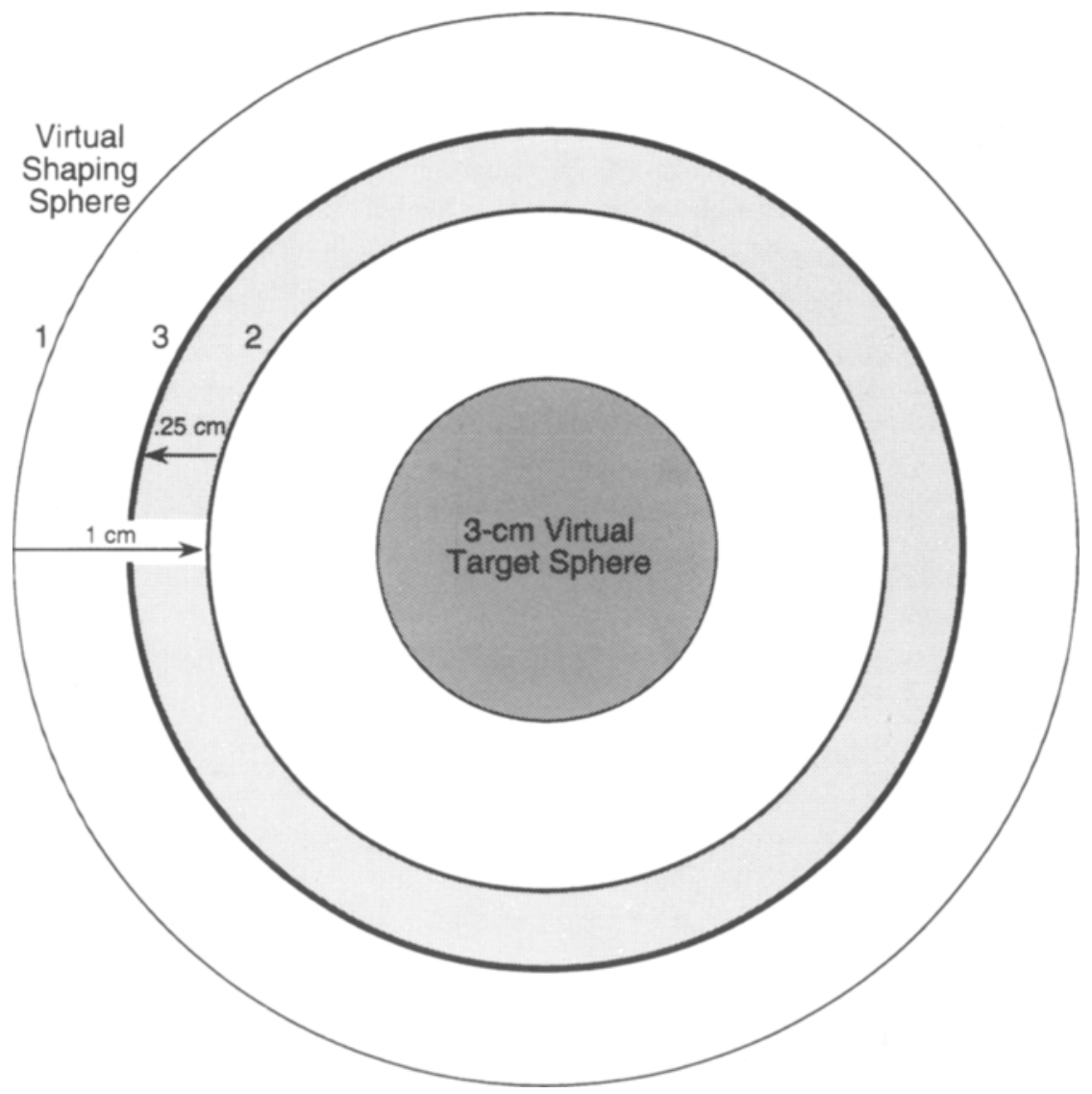

Figure 1. A schematic representation of Pear and Legris's (1987) shaping procedure. The computer program defined a $3-\mathrm{cm}$ virtual target sphere (depicted by the dark gray circle in the diagram). Concentric with the target sphere was a shaping sphere whose radius was programmed to contract by $1 \mathrm{~cm}$ when it was contacted by the bird's head (depicted by the decrease from radius 1 to radius 2 in the diagram). Each contact with the shaping sphere resulted in reinforcement. For every 10 -sec period in which the bird did not contact the shaping sphere, its radius expanded by $0.25 \mathrm{~cm}$ (depicted by the increase from radius 2 to radius 3 in the diagram). This process repeated itself until the bird contacted the target sphere, at which time the shaping sphere was programmed not to expand and reinforcement occurred only when the bird contacted the target sphere. 
target than a smaller one (the first variable from the preceding list). Similarly, a shaping sphere that contracts, say, $10 \mathrm{~cm}$ when contacted by the animal is likely to be more effective than a sphere that contracts $1 \mathrm{~cm}$, but only if the animal is moving swiftly enough to contact the new radius soon after it contacted the previous radius (the second variable in the preceding list). Otherwise, if the animal is moving slowly, it may spend too much time "searching" for the new criteria. But, even for a slowmoving animal, this may be less of a problem if only a brief amount of time is allowed to elapse without a contact with the new radius of the shaping sphere before it begins to expand (the third variable). In this case, the next reward is not too far off; however, there is the possibility that providing too much reward for insufficient progress toward the target will hinder, not help, shaping (Martin \& Pear, 1996). The effect on the shaping process of the amount of time allowed to elapse without a contact with the new radius of the shaping sphere before it begins to expand may be mediated by the distance that the radius of the shaping sphere expands (the fourth variable). In other words, a sphere that expands too far and too soon after the previous reward may hinder successively closer approximations. Finally, if the behavior of the animal is not sampled with sufficient frequency, approximations to the target may go undetected (and unrewarded), potentially retarding the shaping process (the fifth variable). This would be similar to a human observer who was distracted at a moment when a rat he/she was trying to train to leverpress contacted the lever, but was not reinforced for this behavior.

In sum, the five parameters described above, all of which are controlled by the experimenter, may interact with each other and the animal's behavior in ways that affect the shaping process. What follows is a description of a demonstration that allows the user to specify the values of each of these variables to assess their effect on shaping. The demonstration assumes that the instructor has described shaping in sufficient detail that the students understand the potential significance of these variables. Also, though it is not essential, having the students read Pear and Legris's (1987) short article on automated shaping might help them further appreciate the demonstration. SimpleText files containing portions of the present article and instructions on graphing and printing the data are available to help students use the demonstration and understand the significance of the five shaping parameters.

\section{DEMONSTRATIONS OF COMPUTER-CONTROLLED SHAPING}

\section{Method}

The demonstration begins by having a user sit at a Macintosh computer and starting the shaping program written in THINK Pascal 4.0. The program begins by displaying the following set of statements and general instructions.

This program allows you to experience having your behavior shaped, and how different ways of shaping affect how the target response is learned. In this demonstration, the computer will define an invisible circle located on the screen. Your task is to find the location of the circle. The computer will beep when you are getting closer. Once you find the circle, the program will automatically stop, and graphs of your data will be plotted.

You will be prompted to enter the name of the file to which the data will be saved. Next, you will be asked to enter the values of five variables that may be important to the shaping process. These include the (a) size of the radius of the target circle, (b) distance the shaping circle contracts when you contact it, (c) amount of time permitted to pass without an additional contact before the shaping circle expands, (d) distance that the shaping circle expands, and (e) frequency at which the position of the cursor is sampled.

As specified in the instructions, the user is asked to specify the name of the file to which the data will be saved and to enter the values of the shaping parameters. The user is prompted to stay within a specified range of integer values-for example, between 1 and 100 for the radius of the target circle. All distances inputted by the user are in units of pixels (integers only); all times are in units of seconds (or fractions of, because real numbers are permitted).

Once all parameters have been entered, the program begins by clearing the screen and randomly determining the location of the center of the target. The computer then detects the position of the cursor on the screen and sets the radius of the shaping circle to the distance between the cursor and the target. Each contact between the cursor and the shaping circle results in a brief beep (reinforcement). On each succeeding contact between the cursor and the shaping circle, the radius of the circle contracts by the amount specified by the user. For every $x$-sec period (specified by the user) in which contact does not occur, the radius expands by $x$ pixels (again, a distance specified by the user). This process continues until the user contacts the target with the cursor, at which time the demonstration ends and the results are plotted on the screen. Even an "accidental" contact with the target would end the program. ${ }^{2}$ The data in Figures 2 and 3 were generated by the same user; the data in Figures 4,5 , and 6 were generated by three other users. In all cases, the instructor specified the parameters of the shaping procedure.

For this article, the position of the cursor was recorded every $0.2 \mathrm{sec}$ on a Macintosh Plus. The resulting textfile of data contained an array of values for each of the following variables: time, $x$-coordinate of the cursor, $y$-coordinate of the cursor, distance of the cursor from the target, the radius of the shaping circle, and the cumulative number of contacts with the shaping circle (which is also a measure of the number of reinforcers, since all contacts with the shaping circle result in reinforcement). Although the data are plotted on the screen at the conclusion of a session, the textfile of data is comma delineated and can be opened into columns using commercially available software packages, such as Microsoft's Excel, for more sophisticated graphing and analysis. 

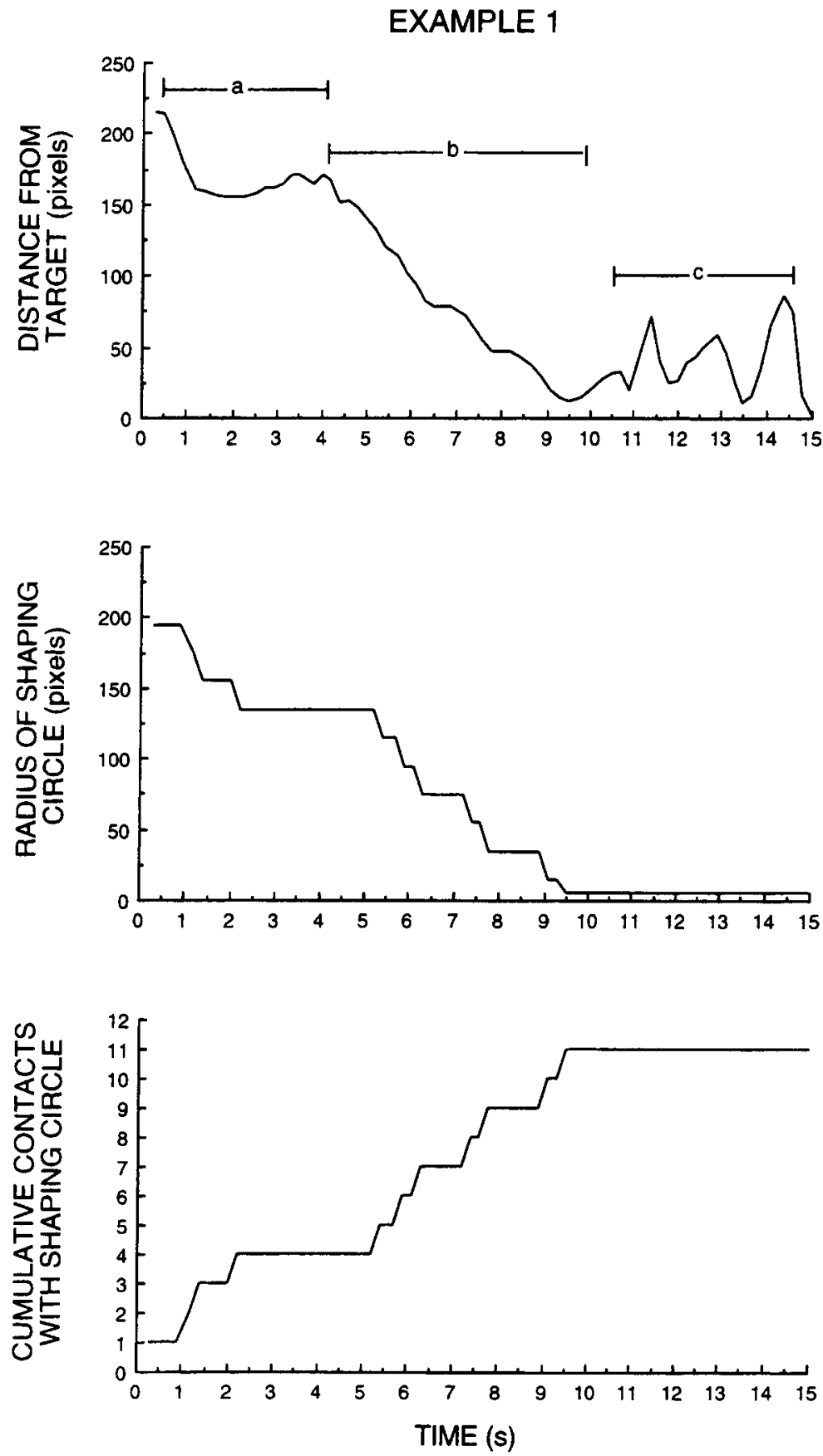

Figure 2. Data from a session in which the radius of the target was 5 pixels, and contacts with the shaping circle caused its radius to contract by 20 pixels. The radius of the shaping circle was programmed not to expand. The top graph shows the distance of the cursor from the target across time; the middle graph shows the radius of the shaping circle across time; and the bottom graph shows the cumulative number of contacts of the cursor with the shaping circle. Because each contact with the shaping circle resulted in reinforcement, the bottom graph also shows the cumulative number of reinforcers.

\section{Examples of Shaping}

The data in Figure 2 are displayed similarly to those plotted by Pear and Legris (1987). The top, middle, and bottom graphs show the distance of the cursor from the center of the target circle, the radius of the shaping circle, and the cumulative number of contacts with the shaping circle (also the cumulative number of reinforcers), respectively. The radius of the target was 5 pixels, and con- 
tacts with the shaping circle caused its radius to contract by 20 pixels. The radius of the shaping circle was programmed not to expand. A summary of the parameters used in all examples described below is presented in Table 1.

Note that the distance of the cursor from the target was greatest at the beginning of the session and then decreased as shaping progressed. However, this decrease in distance was not steady or uninterrupted; three phases of this shaping session are denoted by the sections labeled "a," "b," and "c." In the "a" phase, the cursor moved quickly toward the location of the target (from 0 to $1 \mathrm{sec}$ ), but then failed to make any significant progress until the 4-sec mark. A second phase, labeled "b," began at this point, and was characterized by steady progress toward the target for the next $5.5 \mathrm{sec}$. Pear and Legris (1987) described this as a "ramp" effect. Once near the target, the "c" phase began, which was characterized by lowamplitude oscillatory movements toward and away from the target until it was eventually contacted.

The middle graph shows the radius of the shaping circle across time. Note that, because the shaping circle was programmed not to expand, its radius only decreased or remained constant. In relation to the cursor's distance from the target (top graph), instances in which the radius of the shaping circle remained constant were correlated with movements away from the target. This is especially evident between the 2- and 5 -sec marks and again between the 10- and 15-sec marks.

The bottom graph shows the cumulative number of contacts of the cursor with the shaping circle. Because reinforcement occurred each time the shaping circle was contacted, this graph also shows the cumulative number of reinforcers. Note that this graph is roughly an inverted image of the graph displaying the radius of the shaping circle across time (i.e., the middle graph in the figure).

The graphs in Figure 3 are displayed in the same manner as those in Figure 2. The radius of the target was 2 pixels, contacts with the shaping circle caused its radius to contract by 20 pixels, and failure to contact this new radius within $1 \mathrm{sec}$ caused the radius to expand by 2 pixels. In the top graph, note that the distance of the cursor from the target was greatest at the beginning of the session and then decreased steadily as shaping progressed (i.e., the ramp effect).

Table 1

Summary of Shaping Parameters Used in Each Example

\begin{tabular}{|c|c|c|c|c|c|}
\hline Example & $\begin{array}{l}\text { Radius } \\
\text { of Target } \\
\text { (Pixels) }\end{array}$ & $\begin{array}{c}\text { Contraction } \\
\text { Distance* }\end{array}$ & $\begin{array}{c}\text { Time } \\
\text { Elapsedt }\end{array}$ & $\begin{array}{l}\text { Distance Shaping } \\
\text { Circle Expands } \\
\text { (Pixels) }\end{array}$ & $\begin{array}{l}\text { Sampling } \\
\text { Frequency } \\
\text { (Seconds) }\end{array}$ \\
\hline 1 & 5 & 20 & no limit & 0 & 0.2 \\
\hline 2 & 2 & 20 & 1 & 2 & 0.2 \\
\hline 3 & 5 & 50 & 5 & 50 & 0.2 \\
\hline 4 & 2 & 200 & 5 & 5 & 0.2 \\
\hline 5 & 2 & 20 & 5 & 2 & 0.2 \\
\hline
\end{tabular}

*Distance shaping circle contracts when contacted (pixels). ${ }^{\dagger}$ Amount of time permitted to elapse without a contact before shaping circle expands (seconds).
The middle graph shows that the radius of the shaping circle was steadily driven down toward the radius of the target and that the rate of this decrease was similar to the rate that the distance of the cursor from the target decreased (cf. the slopes of the data in the top and middle graphs). However, unlike in Example 1, failure to contact the new radius within 1 sec caused the radius to expand, though not by as great a distance as the circle contracted. A pattern of two or three small expansions followed by a large contraction is evident in the graph.

The bottom graph of the cumulative number of contacts of the cursor with the shaping circle is an inverted image of the graph of the radius of the shaping circle across time. In fact, the slopes of the data depicted in the top, middle, and bottom graphs are all similar.

The graphs in Figure 4 are from a session in which the radius of the target was 5 pixels, contacts with the shaping circle caused its radius to contract by 50 pixels, and failure to contact this new radius within $5 \mathrm{sec}$ caused the radius to expand by 50 pixels. Unlike the previous two examples, the top graph in Figure 4 shows that the distance of the cursor from the target was greatest about $20 \mathrm{sec}$ into the session, rather than at the beginning. But, as in Example 1, there are at least three distinct phases illustrated in the graph. In the "a" phase, the participant failed to make any significant progress toward the target for the first $30 \mathrm{sec}$; instead, she engaged in a series of oscillatory movements relatively far away from the target. However, for the next $30 \mathrm{sec}(t=30$ to $60 \mathrm{sec})$, the ramp effect is evident as the cursor moved steadily toward the target. After almost contacting the target, the participant engaged in series of high-amplitude oscillatory movements for the next $135 \mathrm{sec}$ (from about $t=60$ to $195 \mathrm{sec}$ ) that moved the cursor toward and away from the target. These oscillations are especially clear in the section labeled " $b$ " in the graph. The last phase, labeled "c," shows that the participant made fewer variable movements, which culminated in contact with the target.

The middle graph shows that the radius of the shaping circle was driven down toward the radius of the target during the first $60 \mathrm{sec}$. Moreover, unlike in Examples 1 and 2 , the radius decreased more abruptly and in a step-like fashion. Once the radius of the shaping circle was equal to that of the target (just before $t=60 \mathrm{sec}$ ), it expanded and contracted at a regular rate for the remainder of the session. Finally, the plot shows that the shaping circle contracted by the same distance that it expanded.

The bottom graph shows a steady rate of contact between the cursor and the shaping circle. Also, unlike the data in the previous two examples, the data depicted in the graph are not an inverse image of the plot of the radius of the shaping circle across time.

The graphs in Figure 5 are from a session in which the radius of the target was 2 pixels, contacts with the shaping circle caused its radius to contract by 200 pixels, and failure to contact this new radius within $5 \mathrm{sec}$ caused the radius to expand by 5 pixels. As in Example 3, the top graph in Figure 5 shows that the distance of the cursor 

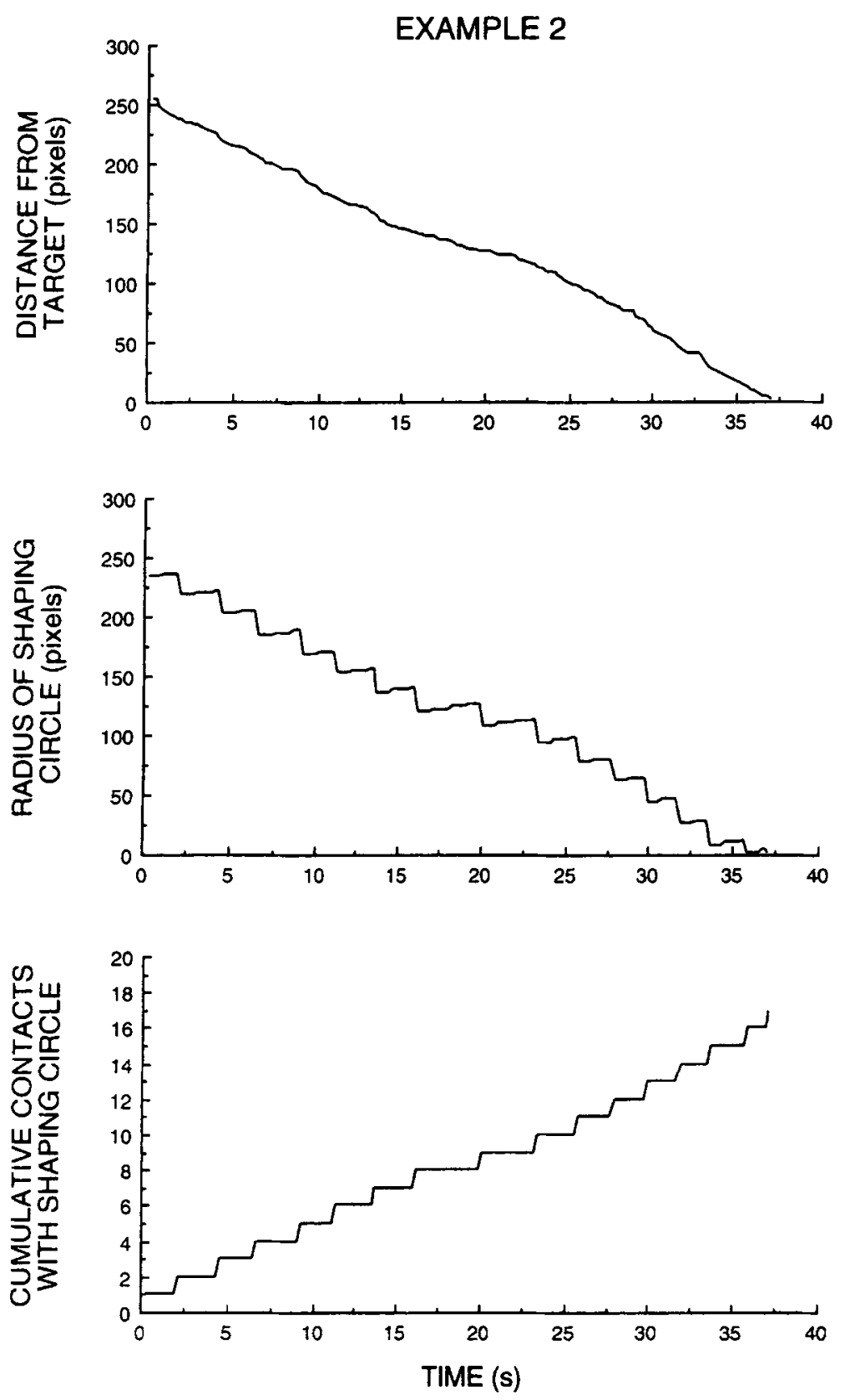

Figure 3. Data from a session in which the radius of the target was 2 pixels, contacts with the shaping circle caused its radius to contract by 20 pixels, and failure to contact this new radius within 1 sec caused the radius to expand by 2 pixels. All other details are the same as in Figure 2.

from the target was greater at several instances during the session than it was at the beginning of the session. More specifically, in the "a" phase, the cursor went progressively farther from the target until it was almost as far as it could be from it, at which time the participant began to move the cursor closer to the target (the end of the "a" phase). Although the cursor almost contacted the target at the 55-sec mark, the participant again moved the cursor farther from the target and repeated this sweeping approach-withdrawal movement again during the " $b$ " phase. This was followed by a series of more restricted movements during the " $\mathrm{c}$ " phase, which culminated in contact with the target.

The middle graph illustrates that, when contacted by the cursor, the radius of the shaping circle contracted more than it expanded. Failure to contact this new radius within $5 \mathrm{sec}$ caused the slow, step-like increases in the size of the shaping circle. The bottom graph of the cumulative 


\section{EXAMPLE 3}
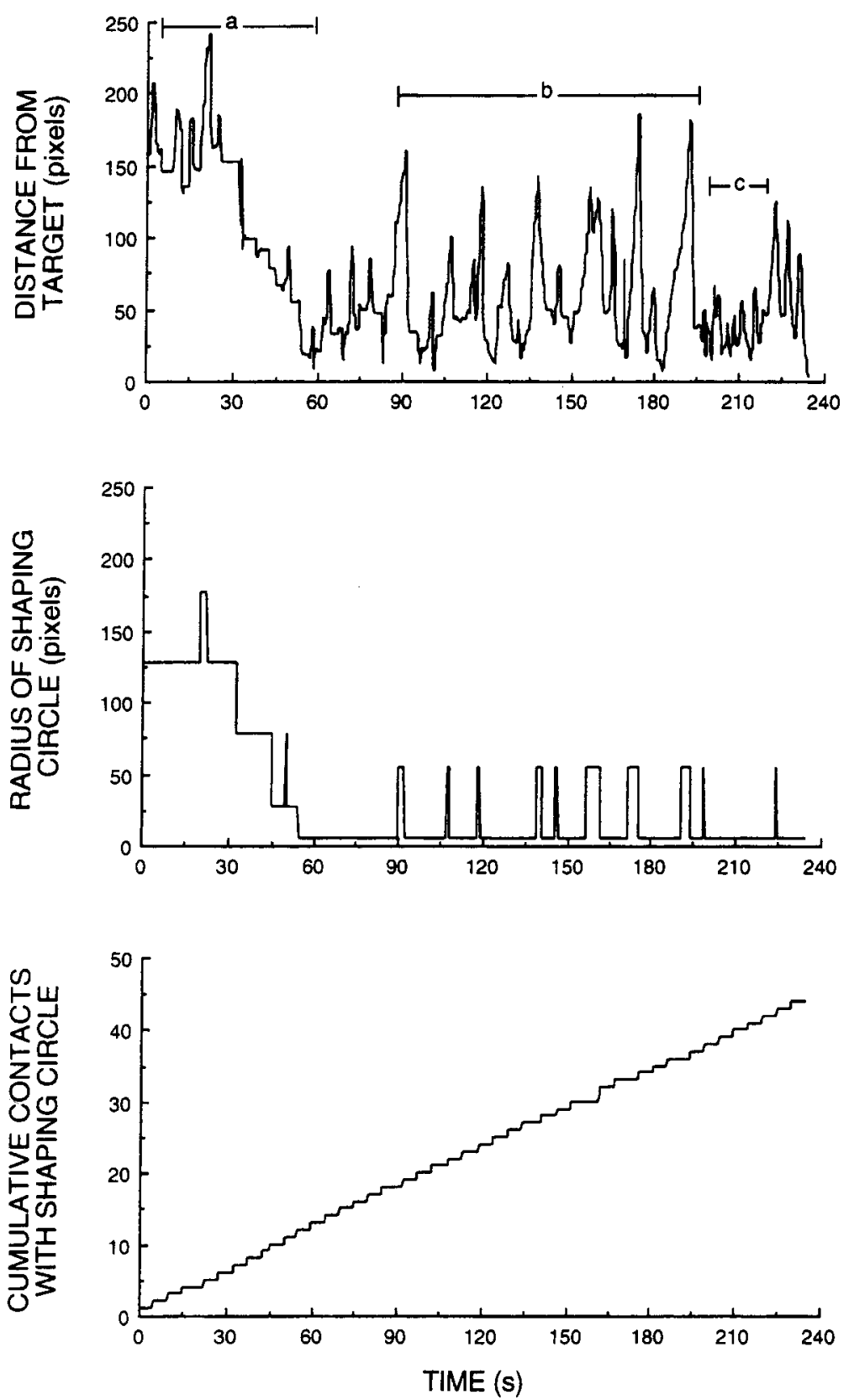

Figure 4. Data from a session in which the radius of the target was 5 pixels, contacts with the shaping circle caused its radius to contract by 50 pixels, and failure to contact this new radius within 5 sec caused the radius to expand by 50 pixels. All other details are the same as in Figure 2.

number of contacts of the cursor with the shaping circle shows some long pauses without a contact, and few total contacts in comparison with the previous examples.

The graphs in Figure 6 are from a session in which the radius of the target was 2 pixels, contacts with the shaping circle caused its radius to contract by 20 pixels, and failure to contact this new radius within $5 \mathrm{sec}$ caused the radius to expand by 2 pixels. As in Examples 3 and 4, the top panel in Figure 6 shows that the cursor was near the target early in the session (the section labeled "a"), but then began to move farther from the target for the next $60 \mathrm{sec}$ (the section labeled " $\mathrm{b}$ "). This movement away from the target consisted of a series of relatively highamplitude oscillatory motions. At the end of the " $b$ " phase, the cursor moved rapidly toward the target. This phase was followed by a series of more restricted movements 

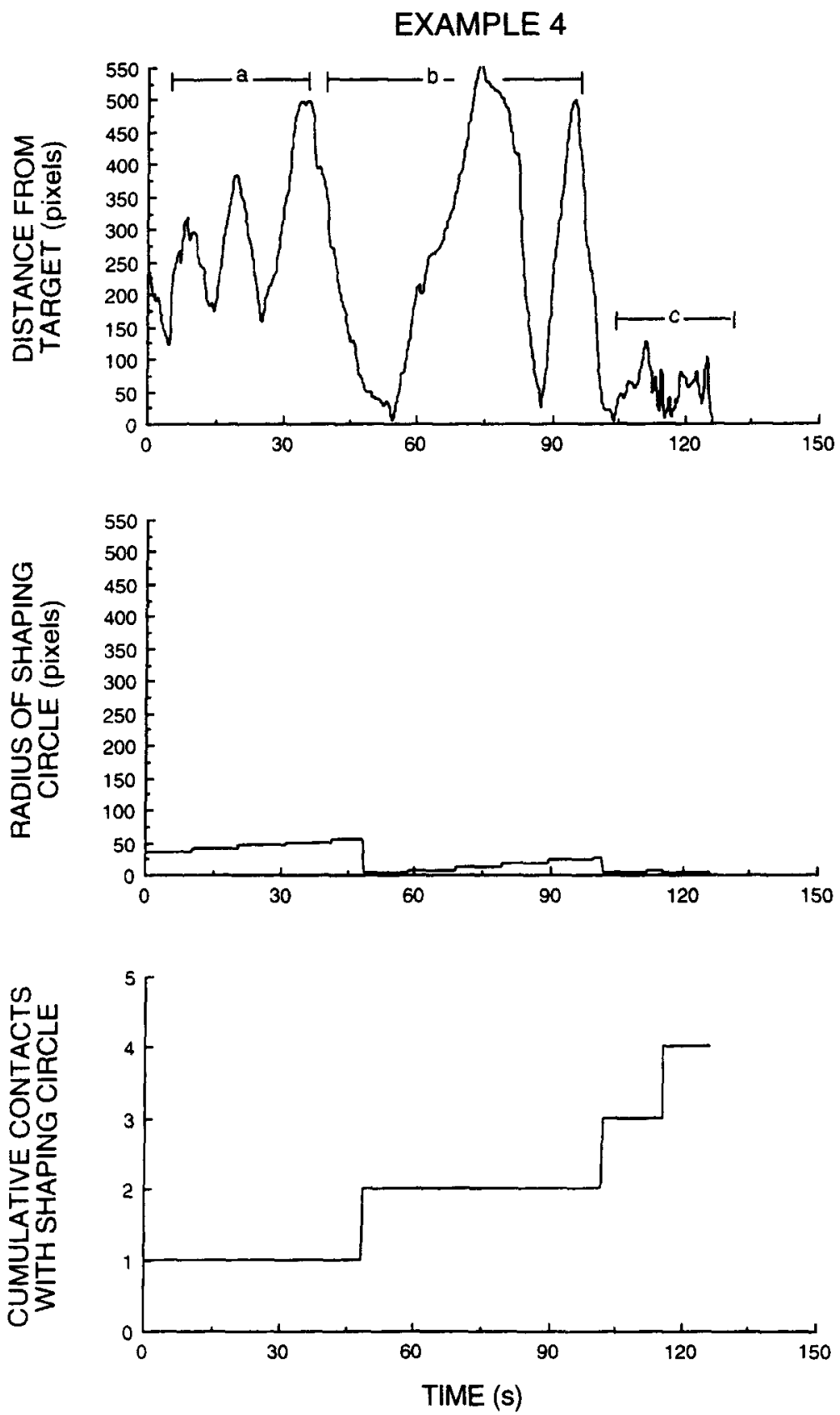

Figure 5. Data from a session in which the radius of the target was 2 pixels, contacts with the shaping circle caused its radius to contract by 200 pixels, and failure to contact this new radius within 5 sec caused the radius to expand by 5 pixels. All other details are the same as in Figure 2.

during the "c" phase, which culminated in contact with the target.

The middle graph shows that the radius of the shaping circle generally expanded across the session until about the 75 -sec mark. At this time, the radius was rapidly driven down to the size of the target. Finally, it can be seen in the plot that the shaping circle contracted a greater distance than it expanded, but the expansions were frequent.

The bottom graph of the cumulative number of contacts of the cursor with the shaping circle shows some long periods without a contact, followed by a high rate of contact beginning at the 75-sec mark. These frequent contacts are correlated with the rapid oscillatory movements near the target (phase " $\mathrm{c}$ " in the top graph) and the rapid shrinking of the shaping circle (middle graph).

\section{DISCUSSION}

This demonstration is an adaptation of Pear and Legris's (1987) technique, which was developed to shape 

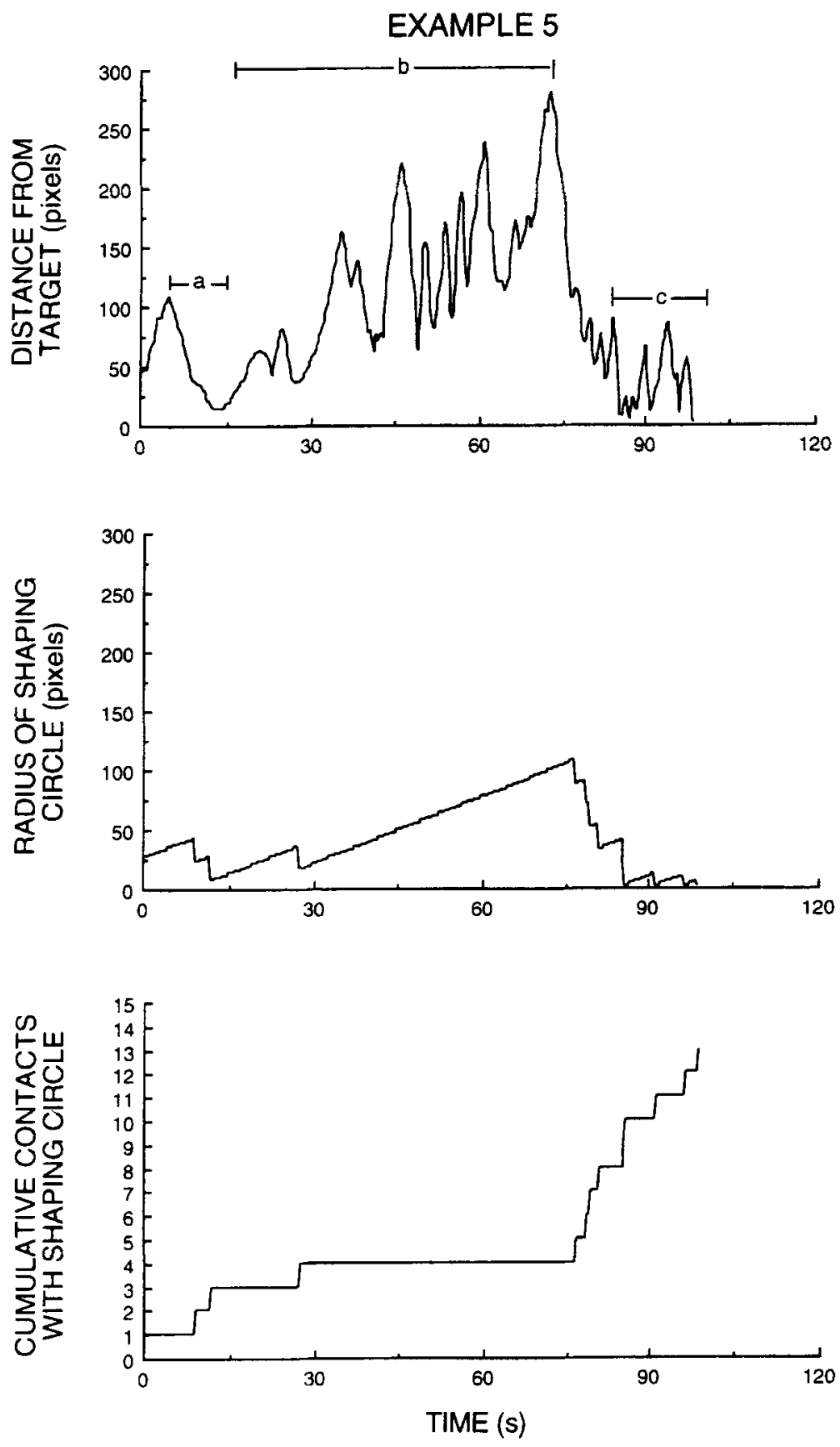

Figure 6. Data from a session in which the radius of the target was 2 pixels, contacts with the shaping circle caused its radius to contract by 20 pixels, and failure to contact this new radius within 5 sec caused the radius to expand by 2 pixels. All other details are the same as in Figure 2.

the movements of pigeons in an experimental chamber. In their study, pigeons were reinforced for contacting a virtual target sphere located near the lower left rear corner of the chamber. However, in the present demonstration, a student sits at a computer and has the position of a cursor shaped by a computer program that reinforces successive approximations to contact with a target location (a virtual circle).

Perhaps the most significant experiential benefit of using the demonstration is that it allows students to feel what it is like to have their behavior shaped. Besides being an inaccurate depiction of shaping, the hot-and-cold game 
is too time-consuming for all students to experience having their behavior shaped. However, the program controlling the present demonstration can be installed on any number of computers in a lab or cluster, and it can accommodate as many users as there are computers.

There also are several important educational benefits of using the demonstration that help students understand shaping and appreciate its challenges. Because the program allows students to systematically manipulate any combination of five variables that may be important to the success of shaping-the size of the target circle, the distance the shaping circle contracts when it is contacted by the cursor, the amount of time allowed to elapse without an additional contact before the shaping circle expands, the distance that the shaping circle expands, and how frequently the position of the cursor is sampled-the students can see how different values of these variables affect shaping. This cannot be done with the hot-and-cold game.

For example, as suggested by Example 3, expanding the shaping circle too far and too often can produce a situation in which the person is reinforced too frequently for an approximation (or, sometimes, for a previous approximation), thereby interfering with the learning of closer approximations (see Martin \& Pear, 1996). Imagine an extreme situation in which the shaping circle expands to fill the computer screen every $5 \mathrm{sec}$ and then contracts to the size of the target. In this situation, there is no need for the person to move the cursor for reinforcement because, whatever his/her behavior, reinforcement will occur at least every $5 \mathrm{sec}$. In fact, this is similar to what pigeons do when the shaping sphere expands and contracts too far every $15 \mathrm{sec}$ - they remain near the feeder waiting for the next reward (see Pear \& Legris, 1987). Experienced shapers know that they have to work within the limits of delivering too much and too little reinforcement for any behavioral approximation (Catania, 1998).

Some difficulties observed in Example 4 might be attributed to reducing the size of the shaping circle too far, leaving the participant searching for the new location. However, the presence of this problem may depend on the person's behavior. To clarify this point, imagine that someone moves the cursor very rapidly. Now consider the following questions: Would shaping be facilitated or hindered if, say, the shaping circle contracted by a relatively short distance? What if the circle contracted by a relatively long distance? Would our answers to these questions change if the person moved the cursor very slowly? In Example 4, the size of the shaping circle likely decreased too far, causing the participant to search for the new radius. However, this might have been less of a problem if the person generally made rapid and sweeping movements, thereby increasing the chances of contacting the new radius of the shaping circle. This example illustrates the importance of selecting an approximation that is relatively easy to attain, but it also highlights a difficulty that may hinder the search for general rules of shaping. Any rules that are eventually discovered may depend on the behavior of the person or animal being shaped. Stated another way, the shaping parameters may interact and alter so drastically the temporal patterns of shaped behavior that this new pattern may not fit well with the shaping parameters, thereby leading to a retardation of learning (see Brehmer, 1992). ${ }^{3}$

Note that Examples 1 and 2 illustrate cases in which shaping progressed smoothly because there was a good fit between the participants' movements and the shaping parameters. A common feature of these examples is that the shaping circle was programmed not to expand (Example 1) or to expand frequently for a short distance (Example 2). It seems that shaping worked best when there was just the right amount of reinforcement for any particular approximation, though there are no explicit rules that specify this amount (Catania, 1998). Too much reinforcement for insufficient progress (Example 3) and requiring too much progress for reinforcement (Example 4) seemed to retard acquisition. In Example 5, the distance that the shaping circle expanded may have been too short given how much time was allowed to pass without a contact between the cursor and the circle. Although the shaping circle expanded by 2 pixels, which is the same value used successfully in Example 2, $5 \mathrm{sec}$ was allowed to elapse before the circle expanded in Example 5, but only $1 \mathrm{sec}$ in Example 2.

Although the purpose of the present paper was to illustrate a computer-controlled shaping technique that may help students understand this important process, there are some additional features of the examples described herein that are worth noting. First, except for Example 2, shaping seems to involve at least two major phases: (1) successively closer approximations, some of which consisted of gross movements, toward the region of the target circle, followed by (2) a series of more restrictive movements near the target that led to contact with the target. This second phase is evident in the sections labeled "c" in Figures 2, 4, 5, and 6. The presence of these two major phases is especially interesting given Pear and Legris's (1987) observation that there were two distinct stages in shaping the behavior of pigeons to contact a virtual target sphere. In their study, the two stages were a result of a pigeon (1) walking toward the region of the target sphere and then (2) lowering its head to contact the target. However, the results of the present demonstration suggest that the shaping process may, more generally, involve a two-stage process - the conditioning of gross movements followed by the conditioning of finer movements - regardless of whether there are two distinct behaviors to condition.

These effects suggest some novel hypotheses that can be examined using more participants and the present program or Pear and Legris's (1987) three-dimensional version. One hypothesis is that there may be an optimal quantitative relationship among the various parameters and the speed of the participant's movements. The search for such a quantitative relationship begins by developing a model and an equation that relates the parameters to each 
other (and the participant's behavior). Perhaps this also can be explored as part of a class discussion on shaping. Some obvious quantitative relationships might emerge from more versus less successful shaping sessions. It might be an engaging challenge for students (and instructors) to discover these relationships.

A second hypothesis is that shaping may progress most smoothly if a multistage algorithm is used. For example, one set of parameters could be used to bring the participant's behavior close to the target, but then another set of parameters could be used to fine-tune the participant's behavior. In the present demonstration, a single set of parameters was used throughout the session. Thus, besides serving as a classroom demonstration that allows students to explore the science underlying the art of shaping (Midgley et al., 1989; Pear \& Legris, 1987), the present program may allow us to examine questions important to our understanding of the shaping process itself.

\section{REFERENCES}

ANTONITIS, J. J. (1951). Response variability in the white rat during conditioning, extinction, and reconditioning. Journal of Experimental Psychology, 42, 273-281.

Brehmer, B. (1992). Dynamic decision making: Human control of complex systems. Acta Psychologica, 81, 211-241.

Catania, A. C. (1998). Learning (4th ed.). Upper Saddle River, NJ: Prentice-Hall.

Donahoe, J. W., Burgos, J. E., \& Palmer, D. C. (1993). A selectionist approach to reinforcement. Journal of the Experimental Analysis of Behavior, 60, 17-40.

Eckerman, D. A., Hienz, R. D., Stern, S., \& Kowlowitz, V. (1980), Shaping the location of a pigeon's peck: Effect of rate and size of shaping steps. Journal of the Experimental Analysis of Behavior, 33, 299-310.

MACHADO, A. (1997). Increasing the variability of response sequences in pigeons by adjusting the frequency of switching between the two keys. Journal of the Experimental Analysis of Behavior, 68, 1-25.

Martin, G. L., \& Pear, J. J. (1996). Behavior modification: What it is and how to do it (5th ed.). Englewood Cliffs, $\mathrm{NJ}$ : Prentice-Hall.

Midgley, M., LEA, S. E. G., \& KirbY, R. M. (1989). Algorithmic shap- ing and misbehavior in the acquisition of token deposit by rats. Journal of the Experimental Analysis of Behavior, 52, 27-40.

PEAR, J. J., \& LEgris, J. A. (1987). Shaping by automated tracking of an arbitrary operant response. Journal of the Experimental Analysis of Behavior, 47, 241-247.

PlatT, J, R. (1973). Percentile reinforcement: Paradigms for experimental analysis of response shaping. In G. H. Bower (Ed.), The psychology of learning and motivation (Vol. 7, pp. 271-296). New York: Academic Press.

STOKEs, P. D. (1995). Learned variability. Animal Learning \& Behavior, 23, 164-176.

Timberlake, W., \& Silva, F. J. (1994). Observation of behavior, inference of function, and the study of learning. Psychonomic Bulletin \& Review, 1, 73-88.

\section{NOTES}

1. If you are interested in seeing how shaping is different from the "hot-and-cold" game, try shaping a student to sit on a table at the front of a classroom with his/her back to the other students. Reinforce successively closer approximations by saying "hot" in a consistent tone and volume; remain silent when the student is getting farther from the desired target response. Then, with a different student, play the hot-andcold game, saying "hot," "hotter," "cold," "colder," and the like as the student gets closer and farther from the target response. Many students have a great deal of difficulty learning to sit on a table with their backs to their peers if you try to shape this behavior, but they more readily learn to do so if you play the hot-and-cold game.

An interesting study that could be done with the computerized shaping procedure described in the present article and Pear and Legris (1987) is to compare the relative effectiveness of a more traditional shaping procedure in which the magnitude of reinforcement is held constant with a procedure in which the magnitude increases as the organism emits closer approximations to the target response.

2 . The likeiihood of an accidental contact varies as a function of the radius of the target. Larger targets will likely result in more accidental contacts than smaller targets. This itself reveals something that psychologists have known about shaping: that it is necessary only when the probability of emitting the target response is low.

3. I thank an anonymous reviewer for drawing my attention to this possibility and to the Brehmer (1992) article.

(Manuscript received October 24, 1997 revision accepted for publication March 17, 1998.) 